\title{
Modelling of mirror mode structures as propagating slow magnetosonic solitons
}

\author{
K. Stasiewicz ${ }^{1,2,3}$ and C. Z. Cheng ${ }^{1}$ \\ ${ }^{1}$ National Cheng Kung University, Tainan, Taiwan \\ ${ }^{2}$ Swedish Institute of Space Physics, Uppsala, Sweden \\ ${ }^{3}$ Space Research Centre, Polish Academy of Sciences, Warsaw, Poland
}

Received: 4 September 2009 - Accepted: 26 November 2009 - Published: 2 December 2009

\begin{abstract}
Cluster measurements in the magnetosheath with spacecraft separations of $2000 \mathrm{~km}$ indicate that magnetic pulsations interpreted as mirror mode structures are not frozen in plasma flow, but do propagate with speeds of up to $\sim 50 \mathrm{~km} / \mathrm{s}$. Properties of these pulsations are shown to be consistent with propagating slow magnetosonic solitons. By using nonlinear two fluid theory we demonstrate that the well known classical mirror instability condition corresponds to a small subset in a continuum of exponentially varying solutions. With the measured plasma moments we have determined parameters of the polybaric pressure model in the region of occurrence of mirror type structures and applied it to numerical modelling of these structures. In individual cases we obtain excellent agreement between observed mirror mode structures and numerical solutions for magnetosonic solitons.
\end{abstract}

Keywords. Interplanetary physics (MHD waves and turbulence) - Magnetospheric physics (Magnetosheath) - Space plasma physics (Nonlinear phenomena)

\section{Introduction}

Mirror mode structures represent large modulations of the magnetic field amplitude $(\delta B / B \sim \pm 50 \%)$ measured by satellites in the magnetosheath and in the solar wind; e.g. Kaufmann et al. (1970); Tsurutani et al. (1982); Schwartz et al. (1996). These structures occur most commonly in regions of significant proton temperature anisotropy, $T_{\perp}>T_{\|}$, in a high-beta plasma, and exhibit anti-correlations between magnetic field $\delta B$ and density $\delta N$ perturbations; see a recent review by Lucek et al. (2005). It is usually thought that they are produced by the purely growing mirror instability that

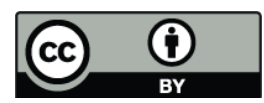

Correspondence to: K. Stasiewicz

(k.stasiewicz@irfu.se) should be excited when the temperature anisotropy satisfies the local instability condition

$\frac{T_{\perp}}{T_{\|}}>1+\frac{1}{\beta_{\perp}}$,

derived in the limit $\omega / k \rightarrow 0$ (Hasegawa, 1969). Here, $\beta_{\perp}=$ $2 \mu_{0} p_{\perp} / B^{2}$ is the perpendicular plasma beta, $\omega$ is the wave frequency and $k$ the wave number. In the above equation, contributions from electrons (much colder than ions in the magnetosheath) and minority ions have been neglected (see e.g. Hellinger, 2007).

A prevalent opinion is that mirror modes represent structures frozen in plasma reference frame that are unstable due to the temperature anisotropy (Southwood and Kivelson, 1993). The instability has been also studied using finite Larmor radius effects (e.g. Pokhotelov et al., 2004; Kuznetsov et al., 2007) and the structures have been the subject of simulation efforts (Baumgärtel et al., 2003; Borgogno et al., 2007; Califano et al., 2008), and analytical studies investigating their relation with slow mode magnetosonic waves (Hau et al., 2005). Recent experimental results based on Cluster measurements include studies by Soucek et al. (2008) and Génot et al. (2009).

The frozen in plasma assumption has been an unverifiable hypothesis before the multi-spacecraft measurements are available. However, the properties of "mirror structures" as observed by Cluster can be quantitatively explained by models showing that they represent propagating trains of slow magnetosonic solitons (Baumgärtel et al., 2003; Stasiewicz, $2004 \mathrm{a}, \mathrm{b}, 2005 \mathrm{a}$ ) moving with speed of $0-30 \mathrm{~km} / \mathrm{s}$, well below the local Alfvén speed. Furthermore, a global model of mirror modes in the bow shock - magnetosheath - magnetopause system by Johnson and Cheng (1997) predicts propagation of these waves due to a combination of diamagnetic drift and the Doppler shift of frequency associated with plasma flow (Johnson and Cheng, 1997). Thus, a reliable determination of the velocity of these structures is important for

Published by Copernicus Publications on behalf of the European Geosciences Union. 
their proper theoretical description. The underlying question is that in case of structures frozen in flow only temperature anisotropy can provide the growth, while in the case of propagation, ion inertia in a moving plasma could be an important factor in mirror mode nonlinear dynamics.

Previous systematic determinations of the velocity of nonlinear structures using Cluster multi-spacecraft measurements (Horbury et al., 2004) were inconclusive because they suffered large errors in measured speeds with mean value of $22 \mathrm{~km} / \mathrm{s}$ and standard deviation of $\pm 33 \mathrm{~km} / \mathrm{s}$. The accuracy of the speed determination depends critically on the separation distance between Cluster spacecraft, which was varied during years of operations with typical separations of $100 \mathrm{~km}$, $1000 \mathrm{~km}$ and $10000 \mathrm{~km}$. With small separations, $\sim 100 \mathrm{~km}$, the short delay times of signals measured by different spacecraft introduce large errors in the determination of the velocity. On the other hand, with large separation distances $\sim 10000 \mathrm{~km}$, the structures observed by different spacecraft have very little or no coherence, which makes impossible determination of their time shifts and velocity. The separation distance of $\sim 1000 \mathrm{~km}$ is found to be ideal for this purpose because the structures are still coherent and the delay time is sufficiently long to determine their velocity accurately.

Using Cluster measurements from the magnetosheath with spacecraft separations of $2000 \mathrm{~km}$ we show that magnetic pulsations interpreted as mirror mode structures are not frozen in plasma, but propagate with speeds of up to $\sim 50 \mathrm{~km} / \mathrm{s}$. Properties of these pulsations are shown to be consistent with propagating slow magnetosonic solitons. These nonlinear solutions of two-fluid equations are sought in the parameter space comprising the wave Alfvén Mach number defined as $M=\left(\omega-\boldsymbol{k} \cdot \boldsymbol{V}_{p}\right) / k V_{A}$, and the propagation angle $\alpha$ with respect to the magnetic field $\mathbf{B}$. Here, $V_{A}$ is the Alfvén speed, and $\mathbf{V}_{p}$ is the bulk plasma velocity in the reference system under consideration. Nonlinear, exponentially varying solutions may occur in different areas of the parameter space $(M, \alpha)$ and in both anisotropic and isotropic plasmas. We demonstrate that mirror instability condition (1) represents a 1-D subset $(M \approx 0)$ in a large area of phase space describing a continuum of exponentially varying solutions. We also show that the polybaric pressure model proposed by Stasiewicz (2005b) represents good approximation to Cluster measurements and can be used in applications related to hot anisotropic plasmas in fluid approximation. Finally, we demonstrate that individual mirror structures could be modelled with high accuracy as slow mode magnetosonic solitons.

\section{Polybaric pressure equations}

A proper model for plasma pressure anisotropy, which may provide free energy for instabilities, is important for mod- elling mirror structures. The anisotropic pressure tensor in the gyrotropic limit can be generally expressed as

$\mathbb{P}=p_{\perp} \mathbb{I}+\left(p_{\|}-p_{\perp}\right) \hat{\mathbf{e}}_{b} \hat{\mathbf{e}}_{b}$,

where $\mathbb{I}$ is the unit tensor, and $\hat{\mathbf{e}}_{b}=\mathbf{B} / B$. For a collisionless and magnetized plasma, Chew-Goldberger-Low (Chew et al., 1956) derived two separate equations for pressure components perpendicular and parallel to the magnetic field $\mathbf{B}$

$p_{\perp} \propto N B, p_{\|} \propto N^{3} B^{-2}$.

The above equations are related to adiabatic invariants of motion and are known as double-adiabatic or CGL model. There have been reservations about the CGL equations because of discrepancies between predictions of kinetic and CGL theories (Abraham-Shrauner, 1967). Some modifications of these equations, consisting essentially on use of different polytropic exponents for perpendicular and parallel pressures, have been proposed by Hau and Sonnerup (1993), and Belmont and Mazelle (1992) who also showed that these exponents depend on the parallel phase velocity in Fourier space, which prevents them being universal.

The pressure equations in collisionless plasma are very important as closure for the fluid hierarchy, and have been the subject of significant efforts in recent years. Notably, anisotropic MHD model that include linear Landau damping was proposed by Snyder et al. (1997). Cheng and Johnson (1999) constructed a kinetic-fluid model that embeds effects of ion Larmor radius (FLR) and wave-particle resonances in the framework of fluid description. More recently, Passot and Sulem (2006) incorporated Landau damping and FLR terms calculated within the gyrokinetic scalings into a fluid hierarchy. Chust and Belmont (2006) have provided a general discussion of the closure of fluid equations in colissionless magnetoplasmas in different limits depending on the parallel wave speed in relation to the thermal speed of particles.

While the above mentioned models properly capture physical problems related to plasma kinetic processes and skillfully attempt to fit them into a fluid hierarchy, they must be specifically adapted to a particular physical problem. A practical solution to this problem has been proposed by Stasiewicz (2005b) who suggested a generalization of CGL equations with exponent parameters derived empirically from space measurements. This model referred to as "polybaric" has four free parameters, without any assumption on adiabaticity

$\begin{aligned} p_{\perp} & =p_{\perp 0}\left(N / N_{0}\right)^{\gamma}\left(B / B_{0}\right)^{\kappa}, \\ a_{p} & =p_{\|} / p_{\perp}=a_{p 0}\left(N / N_{0}\right)^{\gamma_{a}}\left(B / B_{0}\right)^{\kappa_{a}},\end{aligned}$

where $a_{p}$ is the pressure anisotropy parameter and subscript " 0 " denotes background quantities. In a simplified version, with constant anisotropy, $a_{p}$, the model has been used to study soliton solutions, which compare well with Cluster measurements (Stasiewicz, 2004a,b, 2005a), and to study polarization of solitary waves (Mjølhus, 2006). 

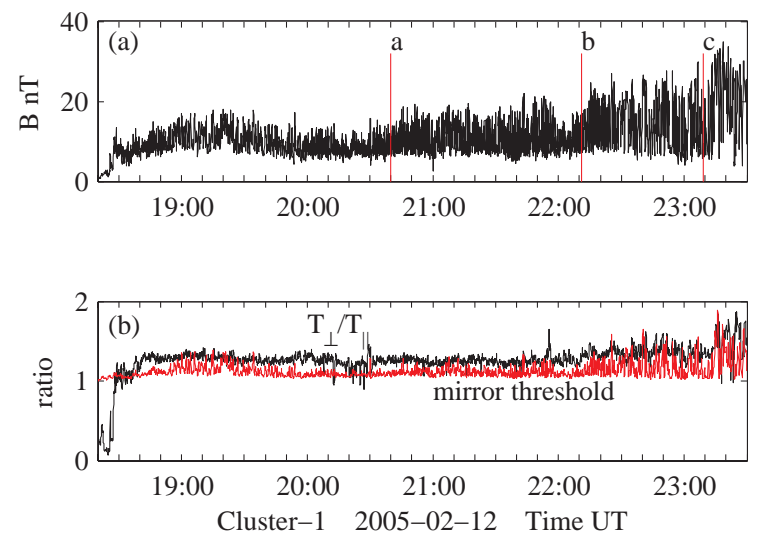

Fig. 1. Cluster observations across the magnetosheath showing (a) magnetic field $B$ and (b) the hot ion temperature ratio $T_{\perp} / T_{\|}$ measured by CIS experiment (black line) together with mirror instability threshold (1), (red line).

To illustrate applicability of this technique we apply it to Cluster measurements made on 12 February 2005 in the magnetosheath on an inbound orbit in the Southern Hemisphere. This is a case with long duration observation of mirror mode structures, shown in Fig. 1 that will be the subjects of detailed analysis in this paper. The spacecraft position was [10.5 - 0.6 - 10.7] $R_{E}$ GSE (Geocentric Solar Ecliptic) at 18:00 UT and [5.1 $-2.5-9.8] R_{E}$ at 23:00 UT. The upper panel shows magnetic field $B$ with a long period of pulsations from 18:30 UT until 23:30 UT, which have properties usually associated with mirror structures in terms of appearance, periodicity, polarization, and propagation direction to the magnetic field. The lower panel shows the temperature anisotropy $T_{\perp} / T_{\|}$as measured by CIS HIA experiment (Rème et al., 2001) and a superposed plot of the mirror instability threshold (Eq. 1). It shows that the plasma was mirror unstable during 18:30-23:30 time interval.

In Fig. 2 we show the polybaric fit to Cluster measurements. A least squares fit of expression (4) to plasma parameters measured by CIS (HIA) gives $p_{\perp} \propto N^{1.45} B^{0.14}$, and $a_{p} \propto 0.8 N^{-0.62} B^{-0.24}$, with overall correlation of 0.90 between the model and measurements. A similar fit for the parallel pressure gives $a_{p 0}=0.8, \gamma_{a}=-0.62$ and $\kappa_{a}=$ -0.24 . Despite its simplicity the model gives valuable insight how plasma perpendicular temperature, $T_{\perp} \propto N^{\gamma-1} B^{\kappa}$, and anisotropy, $a_{p}=T_{\|} / T_{\perp}$, varies with background plasma parameters $N, B$, providing hints on the exchange of energy between particles and fields. For example, in the analyzed case the ion temperature measured by Cluster- 1 behaves like $T_{\perp} \propto N^{0.45} B^{0.14}$, with index $\gamma$ lower than the adiabatic one and with $B$ dependence different from $T_{\perp} \propto B$, as would be expected from conservation of the first adiabatic invariant. An underlying assumption for the fitting of the polybaric expressions (4), (5) is that the data are taken from the same flow streamline, which may not be the case for longer inter-
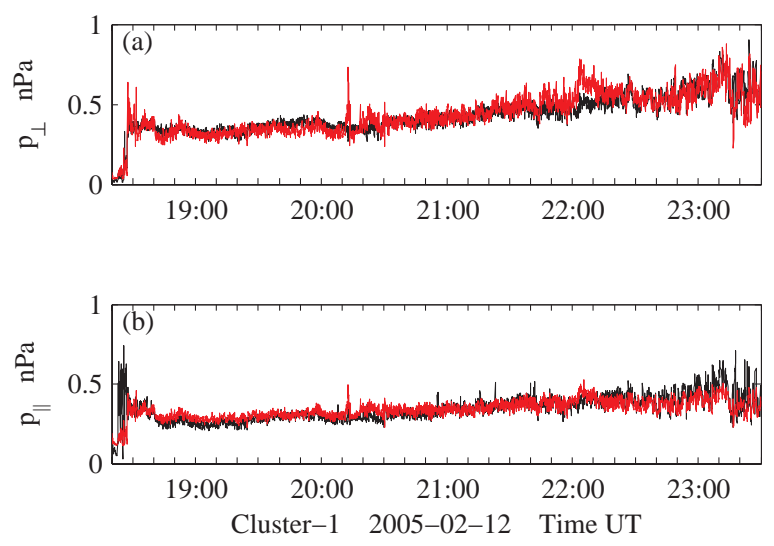

Fig. 2. (a) Perpendicular ion pressure (black line) as measured by Cluster CIS (HIA) experiment in a region of mirror structures. Superposed is fit (red line) with expression (4), which yields $\gamma=1.45$, $\kappa=0.14$. (b) Similar fit for the parallel pressure gives $a_{p 0}=0.8$, $\gamma_{a}=-0.62$ and $\kappa_{a}=-0.24$. The correlation coefficient between model and observations for the perpendicular pressure is 0.90 and for the parallel pressure 0.73 .

vals, such as that in Fig. 2. This could be an explanation for some discrepancies seen in Fig. 2.

\section{Dispersive nonlinear waves in anisotropic plasmas}

Let us introduce the dispersive ion inertial length $\lambda_{i}=$ $V_{A} / \omega_{c i}\left(\omega_{c i}\right.$ is the ion cyclotron frequency), and the total plasma beta $\beta_{\perp}=\beta_{i \perp}+\beta_{e \perp}$. Linearization of standard twofluid equations leads to the dispersion equation for MHD modes that include ion and electron inertia effects, finite plasma beta and pressure anisotropy (Stasiewicz, 2005a). Similar approach to nonlinear phenomena observed in space has been advocated earlier by McKenzie et al. (2004) and Sauer et al. (2003). Generalization of two fluid equations for the ion pressure model with variable anisotropy (5) (see Appendix) gives the following linear dispersion relation applicable for both sinusoidal waves, as well as for exponentially varying instabilities (or nonlinear waves)

$k^{2} \lambda_{i}^{2}=\frac{A(C-A) M_{\|}^{-2}}{1+\left(m_{e} / m_{i}\right)(C-2 A)}$

where $M_{\|}=M / \cos \alpha, m_{e} / m_{i}$ is the electron to ion mass ratio, and

$$
\begin{aligned}
A & =M_{\|}^{2}-1+\frac{\beta_{\perp}}{2}\left(a_{p 0}-1\right), \\
C & =\sin ^{2} \alpha D M_{\|}^{2}-\sin ^{2} \alpha\left(a_{n 0} D+a_{b 0}\right) \beta_{\perp} / 2, \\
D & =\frac{1+\left(\kappa+a_{b 0} \cos ^{2} \alpha\right) \beta_{\perp} / 2}{M^{2}-\left(\gamma+a_{n 0} \cos ^{2} \alpha\right) \beta_{\perp} / 2},
\end{aligned}
$$

$a_{n 0}=a_{p 0}\left(\gamma+\gamma_{a}\right)-\gamma$, and $a_{b 0}=a_{p 0}\left(\kappa+\kappa_{a}-2\right)-\kappa+2$. Sinusoidal waves correspond to $k^{2}<0$, while the reverse 


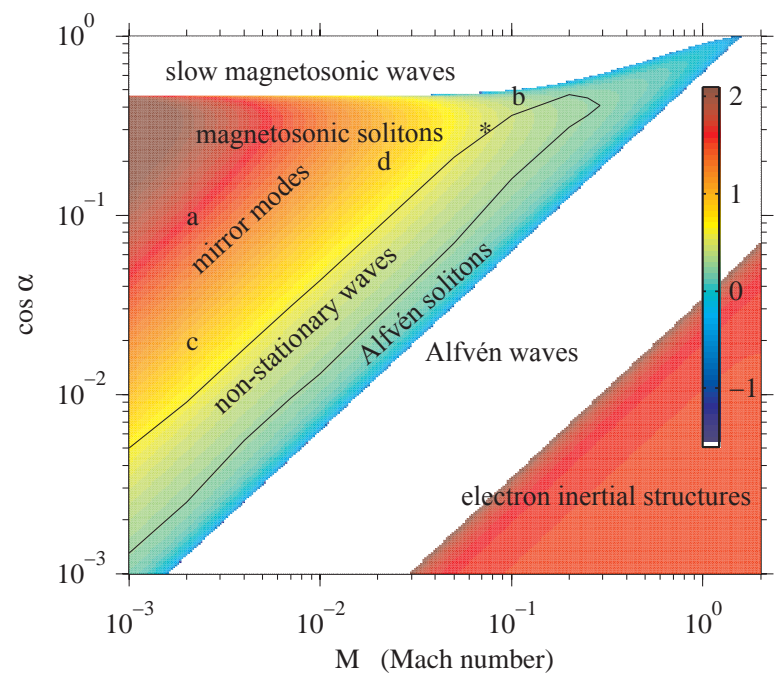

Fig. 3. Phase portrait with growth rate, $\log \left(k \lambda_{i}\right)$ given by Eq. (6), of MHD (including mirror) instabilities in a hot anisotropic plasma for the parameters range from the region of mirror structures observed by Cluster $\left(\beta_{\perp}=11, \gamma=1.60, \kappa=0.10, \gamma_{a}=-0.62, \kappa_{a}=-0.25\right.$, $\left.a_{p 0}=0.714\right)$. Letters a, b, c, d mark locations of solitons shown in Fig. 4. The asterisk "*" shows the location of mirror structure modelled in Fig. 8. The center region is occupied by non-stationary waves.

condition $k^{2}>0$ applies to instabilities (or exponentially varying nonlinear waves).

In the limit of small $\cos \alpha$ and small $M$, the dispersion (6) is expanded as

$$
\begin{aligned}
& k^{2} \lambda_{i}^{2} A^{-1} \approx-1-\frac{2+\kappa \beta_{\perp}}{\gamma \beta_{\perp}}+ \\
& \quad\left(1+\frac{\beta_{\perp}}{2}\left[\frac{a_{n 0}\left(2+\kappa \beta_{\perp}\right)}{\gamma \beta_{\perp}}-a_{b 0}-a_{p 0}+1\right]\right) \frac{\cos ^{2} \alpha}{M^{2}} .
\end{aligned}
$$

It can be seen that exponentially growing solutions $k^{2}>0$ in the limit $M \rightarrow 0$ occur for

$1+\frac{\beta_{\perp}}{2}\left[\frac{a_{n 0}\left(2+\kappa \beta_{\perp}\right)}{\gamma \beta_{\perp}}-a_{b 0}-a_{p 0}+1\right]<0$,

which is equivalent to

$$
\frac{T_{\perp}}{T_{\|}}-1>\frac{1}{\beta_{\perp}}\left(2+\frac{2 \gamma_{a}}{\gamma}\right)+\frac{\kappa \gamma_{a}}{\gamma}-\kappa_{a} .
$$

The above inequality coincides with Eq. (1) for some values of parameters in the polybaric pressure model (Eqs. 4-5), namely for $\gamma_{a}=-\gamma / 2, \kappa_{a}=-\kappa / 2$. Thus, the mirror instability criterium (Eq. 1) derived with kinetic model is contained in the general two-fluid dispersion (6) and the approximation (10) for MHD instabilities, and it corresponds to a one-dimensional region $M \approx 0$ of the two-dimensional $(M, \alpha)$ space. We would like to emphasize that Eq. (6) not only describes mirror modes, but also properly reproduces dispersion equations in the limit of inertial electron Alfvén
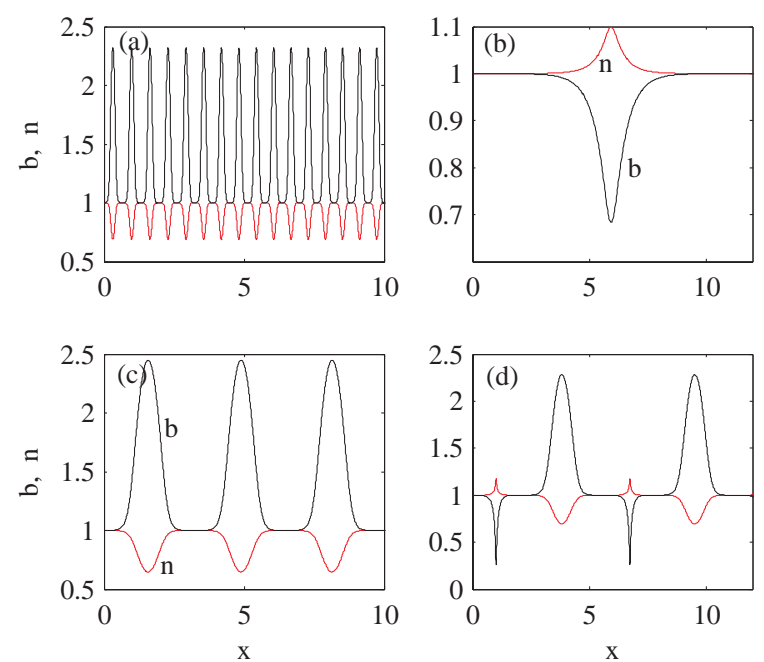

Fig. 4. Examples of soliton solutions in four points marked with letters a, b, c, d in Fig. 3. Black lines show normalized $B$ and red lines normalized $N$. The distance $x$ is in units of the ion inertial length $\lambda_{i}$.

waves (IEAW) and kinetic Alfvén waves (KAW) as shown recently by Stasiewicz and Ekeberg (2008).

To illustrate link between the mirror instability and nonlinear waves we plot in Fig. 3 the linear growth rate of nonlinear waves, $\log \left(k \lambda_{i}\right)$ given by Eq. (6) for parameters corresponding to Cluster observations. It can be seen that in a plasma with pressure anisotropy, exponentially varying solutions exist in large areas between sinusoidal Alfvén waves and slow magnetosonic waves. This region has been denoted as slow alfvenons in the nomenclature introduced recently by Stasiewicz and Ekeberg (2008). Mirror modes, as demonstrated earlier by Stasiewicz (2004a) and reconfirmed further in this paper, belong to a smaller area denoted as slow magnetosonic solitons. An additional red region of unstable waves in the right lower corner corresponds to inertial electron Alfvén wave structures (IEAW), and it would vanish if $m_{e}=0$ is set in Eq. (6); see Stasiewicz and Ekeberg (2008) for details concerning these waves.

The asymptotic behaviour of exponentially unstable solutions $\left(k^{2}>0\right)$ cannot be inferred from the linearized growth rate (Eq. 6) but requires numerical integration of nonlinear equations (A1-A5) given in the Appendix. Such integrations show that the exponentially varying solutions can be divided into two categories: (a) stationary waves (solitons) and (b) non-stationary waves, which do not have integrable solutions. The non-integrable solutions occupy the center part of the colored, slow alfvenon region shown in Fig. 3 and are not discussed in this paper. One can speculate that this area could correspond to observations in the magnetosheath showing chaotic-type nonlinear pulsations, which are actually more common than regular solitons discussed in this paper. 
A large area of the phase space $(M, \alpha)$ has stationary, soliton-type solutions with examples shown in Fig. 4 for points marked with letters a, b, c, d in Fig. 3. There are two types of solitons in this area: Alfvén-type near the border with Alfvén waves and magnetosonic type in the upper part, near the border with slow magnetosonic waves. The difference is mainly in the magnetic polarization $\left(B_{y}, B_{z}\right)$, where $B_{x}$ is in the direction of the minimum variance: it is nearly circular for the Alfvén type and nearly linear (elongated ellipse) for the magnetosonic type. Another important difference is the presence of strong electric field in Alfvén solitons and its absence in magnetosonic solitons.

Mirror mode structures observed in space have nearly linear (or elongated ellipse) polarization, consistent with magnetosonic solitons, as shown elsewhere by Stasiewicz (2004a,b, 2005a). Numerical integrations show that the polarization is controlled mainly by the distance from the instability border with slow magnetosonic waves. It is linear at the border, and becomes more elliptic further out toward the border with non-stationary waves. Both magnetic peaks and magnetic holes solutions can be obtained in unstable areas, consistent with two types of mirror modes measured in space. However, the area with solutions representing magnetic holes is larger than the area with magnetic peaks, an asymmetry which increases with decreasing plasma beta. Furthermore, while the pressure anisotropy greatly increases the area available for alfvenons, they can be also produced in isotropic plasma and in plasma with reversed anisotropy, $T_{\|}>T_{\perp}$, which is important for explanation of similar structures observed in plasma below the threshold (1). In the next section we shall demonstrate that mirror mode structures observed by Cluster do have non-zero speed with respect to plasma, $M>0$, and are consistent with the theoretical model described above.

\section{Cluster measurements and timing analysis}

We now focus on a detailed analysis of magnetic structures measured at times marked with vertical red lines a, b, c in Fig. 1. These time intervals are expanded in Fig. 5, which representatively show soliton-like magnetic pulsations observed by Cluster continuously during four hours. Using the multipoint capabilities of Cluster we shall demonstrate that these structures propagate across the plasma and cannot be regarded as frozen in plasma flow.

\subsection{Determination of the velocity of plasma structures.}

Let us assume the presence of plasma structures with planar fronts moving through space with velocity $\mathbf{V}_{s}$ in the direction normal to the front. These fronts are observed at different
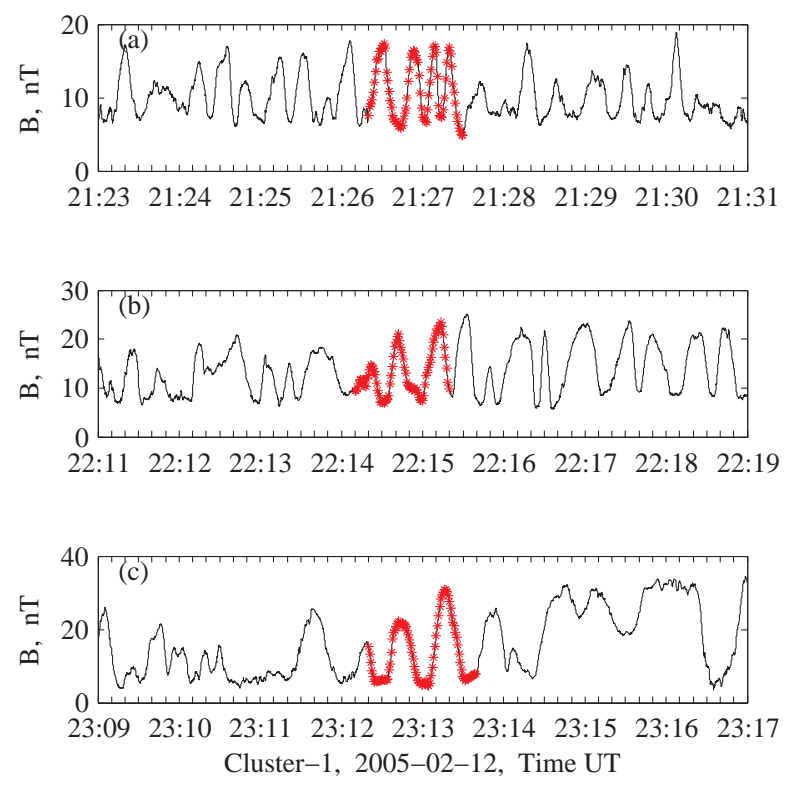

Fig. 5. Zoom at Fig. 1 showing magnetic pulsations in three different times corresponding to labels a, b, c. Notice soliton-like character of the measured pulsations which persists continuously during many hours. Marked intervals are subject to timing analysis in Fig. 6.

times $t_{j(=1,2,3,4)}$ by spacecraft located at positions $\mathbf{r}_{j}$. The relation between these variables is

$\left(\mathbf{r}_{1}-\mathbf{r}_{j}\right) \cdot \frac{\mathbf{V}_{s}}{V_{s}^{2}}=t_{1}-t_{j}$,

which represents three linear equations that can be used to determine velocity $\mathbf{V}_{s}$. A possible transverse velocity in the front plane, $\mathbf{V}_{s t}$, would not contribute to time delays and is undetermined by this method. The normal speed of the structure with respect to the plasma moving with $\mathbf{V}_{p}$ is determined with

$V_{s p}=V_{s}-\mathbf{V}_{p} \cdot \mathbf{n}_{s}$,

where $\mathbf{n}_{s}=\mathbf{V}_{s} / V_{s}$ is the normal vector to propagating planar structures. The above estimate is the minimum speed, which would be larger if the structure is not flowing exactly with $\mathbf{V}_{s t}$. Clearly, all structures measured in space are confined in 3-D. However, if the radius of curvature is much larger than the wavelength or thickness of the structure one can assume local planarity, a procedure commonly used for wave phenomena in space.

In Fig. 6 we show examples of coherent solitary structures measured by all four Cluster spacecraft. Time-series for spacecraft $\mathrm{C} 2, \mathrm{C} 3, \mathrm{C} 4$ have been shifted to align with solitons measured by $\mathrm{C} 1$ and marked with (a), (b), (c), respectively, by maximizing the correlation coefficient. The separation distances between the spacecraft in case "a" are $r_{12}=2279, r_{13}=3040, r_{14}=1737 \mathrm{~km}$, which makes long delay times and reliable velocity determinations. The time 

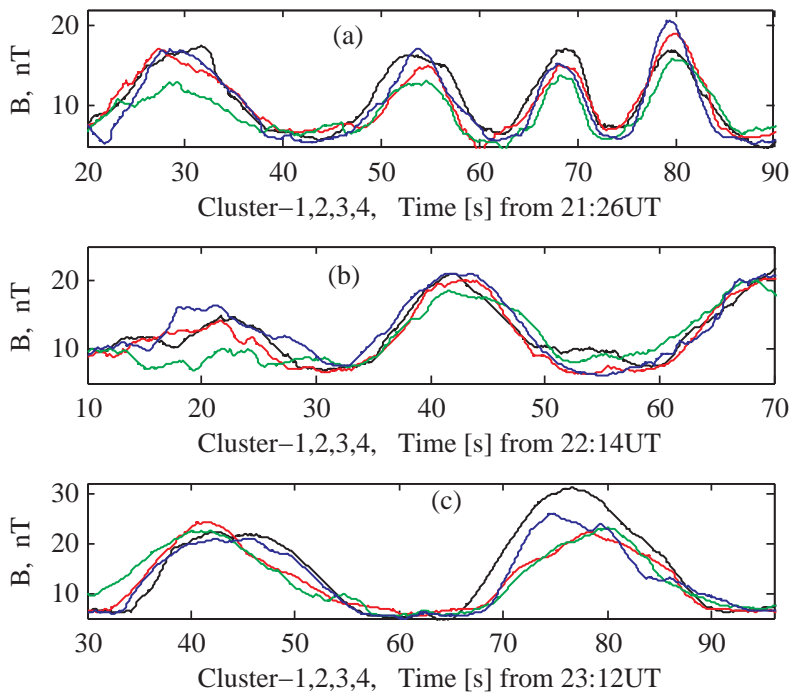

Fig. 6. Magnetic field measured on four Cluster spacecraft shifted in time to align with solitary structures seen by spacecraft $\mathrm{C} 1$ (black) through maximizing the correlation coefficient. The applied time shifts are listed in Table 2.

Table 1. Plasma parameters for time intervals shown in Fig. 6. Ion parameters are averages from CIS-HIA instruments on $\mathrm{C} 1$ and $\mathrm{C} 3$. $\delta V_{p}$ is the standard deviation of the hot ion speed, $V_{p}$.

\begin{tabular}{lrrrrrrr}
\hline event & $\begin{array}{r}N \\
\mathrm{~cm}^{-3}\end{array}$ & $\begin{array}{r}B \\
\mathrm{nT}\end{array}$ & $\begin{array}{r}V_{p} \\
\mathrm{~km} / \mathrm{s}\end{array}$ & $\begin{array}{r}\delta V_{p} \\
\mathrm{~km} / \mathrm{s}\end{array}$ & $\begin{array}{r}V_{A} \\
\mathrm{~km} / \mathrm{s}\end{array}$ & $\beta_{\perp}$ & $\frac{T_{\perp}}{T_{\|}}$ \\
\hline Fig. 6a & 10 & 10 & 248 & \pm 8 & 69 & 11.4 & 1.2 \\
Fig. 6b & 10 & 13 & 217 & \pm 6 & 90 & 7.1 & 1.4 \\
Fig. 6c & 12 & 13 & 159 & \pm 6 & 82 & 9.7 & 1.3 \\
\hline
\end{tabular}

delays between the satellites implied from the alignment of solitons are $t_{12}=12.10, t_{13}=18.00, t_{14}=7.11 \mathrm{~s}$ in the case of Fig. 6a. The computed velocity of the structure is $\mathbf{V}_{s}=[-130$, $25,66] \mathrm{km} / \mathrm{s}$, and $V_{s p}=43 \mathrm{~km} / \mathrm{s}$. The ion flow measured by CIS on C1 and C3 satellites is $\mathbf{V}_{p}=[-197,-23,-145] \mathrm{km} / \mathrm{s}$ and the component perpendicular to the magnetic field is $\mathbf{V}_{p \perp}=[-82,-66,37]$. These quantities are summarized in Table 2 for all three events.

Now, let us assume that structures are aligned with the background field and convect with perpendicular ion velocity $\mathbf{V}_{p \perp}$ as implied by some analyses (Horbury et al., 2004; Constantinescu et al., 2003). We can compute expected time delays from such a configuration using Eq. (13) with substitution $\mathbf{V}_{s} \rightarrow \mathbf{V}_{p \perp}$. These times are $t_{12}=18.9$ (12.1), $t_{13}=24.8$ (18.0), $t_{14}=12.8(7.1) \mathrm{s}$, much different from the observed time delays (in parentheses).
Table 2. Velocity of solitary structures $\mathbf{V}_{s}$ and speed $V_{s p}$ computed with Eqs. (13) and (14) from signal delays derived from multispacecraft timing for three events in Fig. 6. The measured ion velocities, $\mathbf{V}_{p}$, and their perpendicular components $\mathbf{V}_{p \perp}$ are shown for comparison.

\begin{tabular}{lrrrrrrr}
\hline event & $\begin{array}{r}V_{x} \\
\mathrm{~km} / \mathrm{s}\end{array}$ & $V_{y}$ & $V_{z}$ & $V_{s p}$ & $\begin{array}{r}t_{12} \\
\mathrm{~s}\end{array}$ & $t_{13}$ & $t_{14}$ \\
\hline a: $21: 26: 45$ & & & & & & & \\
$\mathbf{V}_{s}$ & -130 & 25 & 66 & 43 & 12.10 & 18.00 & 7.11 \\
$\mathbf{V}_{p \perp}$ & -82 & -66 & 37 & & & & \\
$\mathbf{V}_{p}$ & -197 & -23 & -145 & & & & \\
\hline $\mathrm{b}: 22: 14: 31$ & & & & & & & \\
$\mathbf{V}_{s}$ & -75 & 29 & 32 & 7 & 19.71 & 30.51 & 11.52 \\
$\mathbf{V}_{p \perp}$ & -96 & -96 & 3 & & & & \\
$\mathbf{V}_{p}$ & -164 & -32 & -138 & & & & \\
\hline $\mathrm{c:} 23: 13: 04$ & & & & & & & \\
$\mathbf{V}_{s}$ & -49 & 28 & 1 & -30 & 27.58 & 43.96 & 19.08 \\
$\mathbf{V}_{p \perp}$ & -70 & -63 & -15 & & & & \\
$\mathbf{V}_{p}$ & -102 & -2 & -122 & & & & \\
\hline
\end{tabular}

\subsection{Error analysis}

The numerical results summarized in Table 2 show that mirror mode structures do propagate across the plasma with speeds of up to $43 \mathrm{~km} / \mathrm{s}$ in the analyzed cases, and by no means can they be regarded as frozen in plasma flows. The accuracy of this determination depends on the accuracy in the timing procedure, fluctuations of the ion velocity measured by different spacecraft, and generally on the spatial geometry of the structures and on spacecraft configuration.

The velocity of the structure, $\mathbf{V}_{s}$, determined with Eq. (13) is affected only by the accuracy of the timing $t_{1}-t_{j}$, because the positions of the spacecraft are known with very high accuracy. Time delays for signals presented in Figs. 6 and shown in Table 2 are determined by maximizing the correlation function of two timeseries. The position of the maximum of the correlation function is found with the accuracy corresponding to the sampling time of the magnetometer. However, different time intervals taken for the correlation procedure would produce slightly different time delays. This experience shows that $\delta t \approx 1 \mathrm{~s}$ can be regarded as a typical error in timing. The sensitivity of expression (13) to errors in timing $\delta t$ can be easily checked by substituting different times. The result of such simulations is that $1 \mathrm{~s}$ difference in time delays produces typically $\sim 1 \mathrm{~km} / \mathrm{s}$ difference in $\mathbf{V}_{s}$ components. This applies to the current spacecraft configuration and it would be different in other cases. Thus the errors of timing produce velocity errors that are comparable to errors introduced by the speed of the Cluster s/c, which is $\approx 3 \mathrm{~km} / \mathrm{s}$.

The speed of the structures in the plasma frame given by Eq. (14) is affected by inaccuracies in the determination of ion speeds. As seen in Table 1 the ion speed fluctuates with $\delta V_{p} \approx 10 \mathrm{~km} / \mathrm{s}$, which is the major error entering $V_{s p}$ given by Eq. (14). This error is reduced by the cosine of the 
angle between $\mathbf{n}_{s}$ and $\mathbf{V}_{p}$. In the analyzed cases this angle is $\approx 60^{\circ}$, corresponding to a reduction factor of 0.5 . Thus, $\delta V_{p} \approx 10 \mathrm{~km} / \mathrm{s}$ would produce $5 \mathrm{~km} / \mathrm{s}$ error in $V_{s p}$. Furthermore, the angular inaccuracy of the ion velocity vector corresponding to the angular resolution of $6^{\circ}$ for CIS instrument would produce errors of $\left(1-\cos 6^{\circ}\right) V_{p}$, which amounts to $1.2 \mathrm{~km} / \mathrm{s}$ for ion speeds $V_{p} \approx 200 \mathrm{~km} / \mathrm{s}$.

In summary, the error in the normal velocity of the structure (13) should be not larger than $3 \mathrm{~km} / \mathrm{s}$, while the error in the normal speed in respect to plasma (14) should be not larger than $10 \mathrm{~km} / \mathrm{s}$. Yet another errors could be produced by possible non-planarity of the structures. The planarity of the structures can be estimated using minimum variance analysis (MVA) of the magnetic field. The MVA applied to magnetic field vector in individual structures measured on a single spacecraft gives orthogonal directions where $(x, y, z)$ correspond to minimum, intermediate and maximum variation of $\mathbf{B}$ with eigenvalues $\lambda_{x}, \lambda_{y}, \lambda_{z}$ (Sonnerup and Cahill, 1967). The eigenvector related to the minimum eigenvalue is a proxy to the normal vector $\mathbf{n}_{s}$.

The MVA is applied to single solitons from Fig. 6a, b, c. For time interval 23:13:04-35 in Fig. 6c and spacecraft C3 we obtain the normal vector $\mathbf{n}_{s 3}=[-0.92,0.35,0.16]$ and the ratio of eigenvalues $1: 3: 254$. The normal vectors determined for spacecraft $\mathrm{C} 1, \mathrm{C} 2, \mathrm{C} 4$ differ from that of $\mathrm{C} 3$ by angles $\delta \theta=22^{\circ}, 11^{\circ}, 7^{\circ}$, respectively. Comparison of the directions of $\mathbf{n}_{s 3}$ and of the velocity $\mathbf{V}_{s}$ determined from the spacecraft timing and shown in Table 2 (case c) gives an angle of $12^{\circ}$, which means that the direction of the propagation front determined from spacecraft timing is in a reasonable agreement with the direction determined from MVA. It is a rather known fact that these two methods give somewhat different angles, as shown also in a statistical study of plasma discontinuities in Cluster data by Knetter et al. (2004).

The angle between the velocity of the structure $\mathbf{V}_{s}$ and the perpendicular velocity of ions shown in Table 2 (case c) is $71^{\circ}$. For the total ion velocity this angle is $56^{\circ}$. This means that the popular view of mirror structures convecting with ion flows is not supported by Cluster measurements. The distance between $\mathrm{C} 1$ and $\mathrm{C} 3$ is $r_{13}=3427 \mathrm{~km}$, which implies the radius of curvature $r_{n}=r_{13} / \delta \theta_{13} \approx 9000 \mathrm{~km}$. The observed duration of the soliton $18 \mathrm{~s}$ and speed of $56 \mathrm{~km} / \mathrm{s}$ (Table 2) gives thickness of $1000 \mathrm{~km}$, much smaller than the radius of curvature. A curved front of a structure would introduce additional timing errors. Assuming spacecraft separation of $1000 \mathrm{~km}$ in the plane transverse to the normal direction, and the curvature radius of $9000 \mathrm{~km}$, we find the distance between plane and curved surfaces to be $55 \mathrm{~km}$. A soliton moving through space with speed $56 \mathrm{~km} / \mathrm{s}$ needs $1 \mathrm{~s}$ to cover such a distance. This would introduce additional timing errors of $\delta t \approx 1 \mathrm{~s}$, that would cause errors of $1 \mathrm{~km} / \mathrm{s}$ in $\mathbf{V}_{s}$, as discussed above. An uncertainty of the normal direction of $15^{\circ}$ could produce an error in $V_{s p}$ amounting to $\left(1-\cos 15^{\circ}\right) V_{p}$, which is $8 \mathrm{~km} / \mathrm{s}$ for ion speeds $V_{p} \approx$ $200 \mathrm{~km} / \mathrm{s}$.
Repeating the above outlined MVA procedure for the case in Fig. 6b, time interval 22:14:31-54, we obtain the normal direction $\mathbf{n}_{s 4}=[-0.92,0.13,0.36]$ and the ratio of eigenvalues 1:2:157 for spacecraft $\mathrm{C} 4$. This direction differs from the direction determined from spacecraft timing (Table 2, case b) by $13^{\circ}$. The normal directions determined with MVA with other spacecraft differ from that of $\mathbf{n}_{s 4}$ by $\approx 20^{\circ}$ implying a curved propagating front, similar as in case (c) discussed above. Again, the front direction make large angles $\approx 56^{\circ}$ with perpendicular ion velocity, inconsistent with the assumption of structures being frozen in plasma.

For the case in Fig. 6a, time interval 21:26:45-62, we obtain the normal direction $\mathbf{n}_{s 1}=[-0.78,0.45,0.42]$ and the ratio of eigenvalues $1: 3: 35$ for spacecraft $\mathrm{C} 1$. This direction makes angle of $17^{\circ}$ to the front velocity determined with spacecraft timing and shown in Table 2 (case a). The normal directions determined for spacecraft $\mathrm{C} 2, \mathrm{C} 3, \mathrm{C} 4$, differ from $\mathrm{C} 1$ by angles $\delta \theta=13^{\circ}, 8^{\circ}, 21^{\circ}$, respectively.

The above discussion indicates that the mirror structures propagate across the plasma with speeds determined here of up to $43 \mathrm{~km} / \mathrm{s}$ with error bars of up to $15 \mathrm{~km} / \mathrm{s}$. Their thickness is $\sim 1000 \mathrm{~km}$, and radius of curvature is $\sim 10000 \mathrm{~km}$. The minimum speeds of structures with respect to plasma obtained here $\left(V_{s p} \approx 43,7,-30 \mathrm{~km} / \mathrm{s}\right.$; see Table 2$)$, fall in the range of velocities $-60<V_{s p}<60 \mathrm{~km} / \mathrm{s}(M<0.5)$ obtained earlier from a larger statistical study made by Horbury et al. (2004). Also their determination of propagation angles cover $60-90^{\circ}$, which corresponds to $\cos \alpha<0.5$ and means that their data points would scatter within the colored alfvenon region shown in Fig. 3. These authors used Cluster orbits with small separation between spacecraft, which resulted in large errors in speed determination. Their velocity measurement has a mean value of $22 \mathrm{~km} / \mathrm{s}$ and standard deviation of $33 \mathrm{~km} / \mathrm{s}$ due to short spacecraft separation distance. However, Horbury et al. (2004) incorrectly concluded that their results are consistent with structures being stationary in the plasma frame. In fact, measurement errors do not permit one to claim that the physical values less than the measurement error are equal to zero.

\subsection{Modelling of individual structures}

The position on the diagram in Fig. 3 determines the properties of a soliton. The Mach number $M$ and the propagation angle $\alpha$ must be determined by the boundary conditions or the driver of these structures. The maximum compression of $b=B / B_{0}$ is controlled by the momentum Eq. (A1), which describes a functional relationship between $b$ and $n$. An example of this function is shown in Fig. 7 for two plasma beta $\beta_{\perp}=2$ and 11. All stationary solutions must lie on such curves, which represent typical behaviour of slow modes with anticorrelation between $b$ and $n$, and bi-modal nature of solutions where either magnetic peaks or dips are permitted. Maximum amplitude of $b$ in mirror modes, and generally in all slow mode solitons is controlled mainly by plasma $\beta$. For 


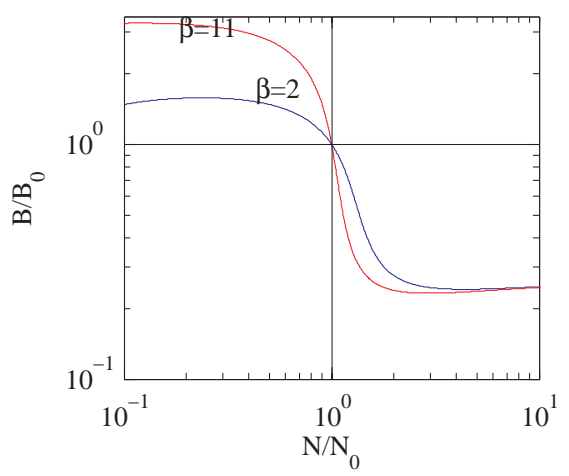

Fig. 7. Relation between the density $n=N / N_{0}$ and the magnetic field $b=B / B_{0}$ implied by Eq. (A1), computed for two betas: $\beta_{\perp}=11$ (red) and $\beta_{\perp}=2$ (blue) lines. Other parameters are: $M=0.1$, $\cos \alpha=0.27, \gamma=1.60, \kappa=0.10, \gamma_{a}=-0.62, \kappa_{a}=-0.25, a_{p 0}=0.714$.

example, in Fig. 7, maximum field compression for $\beta_{\perp}=11$ is 3.2, while for for $\beta_{\perp}=2$ is only 1.5. Parameters $\gamma$ and $\kappa$ in the pressure model determine the steepness of curves shown in Fig. 7, while pressure anisotropy parameters $\gamma_{a}$, and $\kappa_{a}$ affect only shape of $b-n$ curves in the region of magnetic dips.

We have selected the third soliton measured by Cluster2 (red) in Fig. $6 \mathrm{~b}$ for a detailed modelling with the present theory. The minimum variance analysis applied to this case makes it possible to estimate propagation angle of the soliton from the relation $\cos \alpha=\left\langle B_{x}\right\rangle /\langle B\rangle$, where $x$ is the direction of the minimum variance of $\mathbf{B}$. This angle is found to be $\alpha \approx 74^{\circ}$. The Mach number of the soliton was then varied to obtain solution with the same maximum amplitude $B_{\max } / B_{0} \approx 2.7$ as in Cluster observations. Comparison of the modelling results (red " + ") are shown together with Cluster measurements (black lines) in Fig. 8. The measured field magnitude was normalized with the background value $B_{0}=7.1 \mathrm{nT}$. The time axis for Cluster measurements has been converted to spatial scale by free adjustment. The shape and polarization of the soliton compares rather well with observations shown also in Fig. 8.

\section{Discussion}

In this section we briefly discuss predictions of the present soliton model and put them in the context of other models, when applicable.

\subsection{Size of the structures}

Generally, the soliton models with Hall-type dispersion in the generalized Ohm's law have the ion inertial length $\lambda_{i}=$ $V_{A} / \omega_{c i}$ as the major dispersive parameter. The electron inertia terms do not affect the solutions in the $(M, \alpha)$ range of mirror modes. The kinetic models based on FLR effects
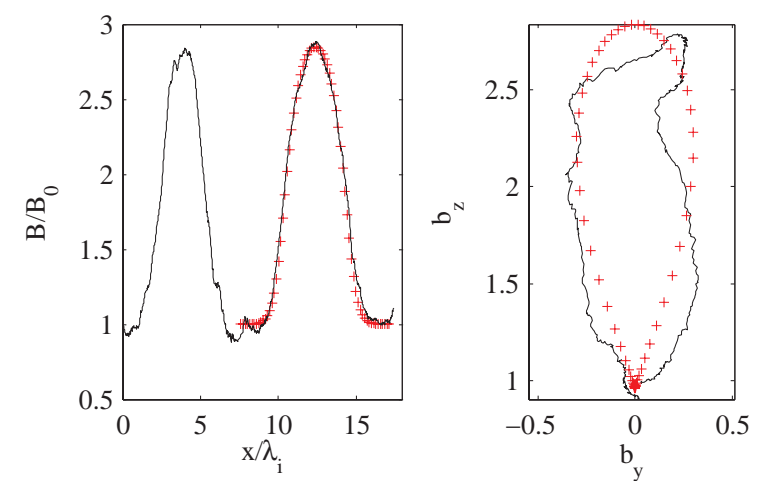

Fig. 8. Comparison of solitons measured by Cluster-2 at time 22:14:10-22:15:05 UT with a numerical solution computed for parameters in Fig. 7, with $\beta_{\perp}=11$, and $M=0.067$. This solution corresponds to the position marked with asterisk in Fig. 3. The computed amplitude and polarization are plotted with red "+".

have the ion gyroradius $r_{i}=V_{t i} / \omega_{c i}$ as the dispersive parameter. These two are related through ion beta as $r_{i}=\sqrt{\beta_{i \perp}} \lambda_{i}$. In the case of Fig. 6c, the solitary structures have observed duration of $18 \mathrm{~s}$ and speed determined with inter-spacecraft timing of $56 \mathrm{~km} / \mathrm{s}$, which gives the size of $1000 \mathrm{~km}$. The sizes obtained with integration depend roughly on the position $(M, \alpha)$ in Fig. 3, and become smaller in red regions and larger in blue regions. They would also depend on $\beta$ and anisotropy $a_{p}$ that affect instability patterns and the area shown in Fig. 3. Generally, the present model predicts and can explain various sizes of fluid-type solitons observed in space and related to ion inertia. Note that estimations of the size of mirror structures assuming that they are frozen in ion flows would give their sizes much larger than sizes estimated from accurate multi-spacecraft timing.

\subsection{Magnetic polarization}

The minimum variance direction is used to determine the propagation angle with respect to the magnetic field, $\cos \alpha=$ $\left\langle B_{x}\right\rangle /\langle B\rangle$, while $\left(B_{y}, B_{z}\right)$ determine the transverse polarization pattern. It may vary from nearly linear for eigenvalues $\lambda_{y} \ll \lambda_{z}$, (equivalently $\left\langle B_{y}\right\rangle \ll\left\langle B_{z}\right\rangle$ ) to quasi elliptic when these inequalities are not fulfilled. Génot et al. (2009) have noted that mirror modes are more commonly observed as elliptically than linearly polarized, contrary to predictions of the linear theory of instability. The present model explains such behaviour, because the polarization pattern depends mainly on the distance form the border with sinusoidal magnetosonic waves in Fig. 3. The polarization is nearly linear at the border and the component $B_{y}$ becomes larger when the soliton solutions approach the non-stationary boundary in Fig. 3. 


\subsection{Amplitude of pulsations and the saturation mechanism}

The early models of saturation processes involved cooling of trapped particles in magnetic troughs (Kivelson and Southwood, 1996; Pantellini, 1998) are applicable mainly to magnetic holes. More recent models involve saturation due to local variation of the ion Larmor radius (Pokhotelov et al., 2004; Kuznetsov et al., 2007). Nonlinear dynamics of mirror modes has been recently reviewed by Califano et al. (2008), who concluded that the saturation mechanism remains a major unresolved problem. The model presented in this paper implies that the saturation mechanism should be related to the mechanism responsible for creation of solitons, i.e. a balance between dispersive and nonlinear terms in the governing Eqs. (A1-A5), constraint by constants of motion. Depending on the initial position of the perturbation in the $(M, \alpha)$ space of Fig. 3, this balance could be achieved at different amplitudes, leading to solitons with a variety of scales and amplitudes. The maximum possible amplitude of $B$ is determined by the normal momentum conservation Eq. (A1), which describes a path in the $(B, N)$ space (see Fig. 7), where physical solutions must follow (Stasiewicz, 2004a,b). Plasma beta is the most important parameter that controls maximum amplitude of $B$ in such curves. Numerical solutions show that for a given propagation angle $\alpha$, the amplitude of the soliton increases with $M$ until it reaches the non-stationary boundary, where apparently a balance between dispersion and nonlinearities cannot be achieved. These non-stationary solutions could correspond to nonlinear chaotic fluctuations observed frequently in the magnetosheath.

\subsection{Growth rate and spatial variation of the amplitude}

A view promoted by some researchers is that the spatial variation of the amplitude of pulsations seen in Fig. 1 indicates that mirror structures generated at the bow shock grow while being convected to the magnetopause. A spatial gradients of amplitudes on large scales $>20000 \mathrm{~km}$ could then be used to determine the growth rate of mirror modes (Tatrallyay et al., 2008). This seems to be an incorrect interpretation because the mirror structures grow on the distances much smaller, comparable to their spatial scales (few $\lambda_{i}$ ) while they propagate. Spatial variations of soliton amplitudes on scales $>20000 \mathrm{~km}$ depend rather on variations of plasma parameters that control the maximum amplitude of the soliton and should not be mixed with their growth rates. Lack of coherence between some solitons seen on different spacecraft in Fig. 6 and in many other cases (not shown here) with similar inter-spacecraft separation indicate that these structures grow and decay on scales of a few thousand $\mathrm{km}$.

\subsection{Two modes of mirror structures: peaks and dips, and their occurrence below the threshold}

Two modes of mirror structures: peaks and dips, the preferred occurrence of dips in lower beta plasma (Joy et al., 2006) and frequently in plasmas below the instability threshold (Soucek et al., 2008; Génot et al., 2009) make big challenge for researchers having in mind non-propagating structures self-created by the instability (1). To circumvent these problems, modifications based on FLR effects have been proposed recently by Kuznetsov et al. (2007) involving what they call as "subcritical bifurcation". The present model has straightforward answers to all these problems: Magnetic peaks and dips represent both normal modes of Hall-MHD system and thus represent two types of asymptotic stationary states. They come naturally from integration of nonlinear Eqs. (A1-A5) as shown in Fig. 4, without involving any special FLR effects. With decreasing of plasma beta the area of integrable solutions with dips (in diagrams like Fig. 3) becomes larger than the area with peaked solutions. This could explain the observed preference of dips in lower beta plasma $(\beta \sim 1)$. Nevertheless it would be worthwhile to find a variational principle which determines why particular types of solutions are selected in different regions. Finally, in lower beta plasmas there is still small area with nonlinear solutions even for isotropic plasma. This can explain why mirror modes (magnetosonic solitons) are observed sometimes well below the threshold (1). Generally, occurrence of mirror modes below the threshold (1) indicates that the ion inertia and soliton behaviour at $M>0$, is the relevant mechanism rather than the temperature anisotropy and mirror behaviour at $M=0$.

\subsection{Mechanism for the generation}

A common view is that mirror structures are initiated at the bow shock and then slowly evolve and grow while being frozen in plasma and convected toward the magnetopause, specifically to regions with increased ratio $T_{\perp} / T_{\|}$. This implies their longevity and a possibility of identification of individual mirror structures on large distances, at least $10000 \mathrm{~km}$. However, from our experience, at Cluster spacecraft separation of $10000 \mathrm{~km}$ the structures do not exhibit much coherence between the spacecraft and it is rather difficult to find a single event good for timing analysis. This indicates that they may not travel so far and models proposing their transport across the magnetosheath may not be valid. The present theory implies an alternative scenario. As can be seen in Fig. 1, pressure anisotropy increases in the magnetosheath from the bow shock toward the magnetopause. Magnetosonic perturbations generated at a given point $(M, \alpha)$ that lies in the sinusoidal (white) area of Fig. 3, and moving toward a region with increasing $T_{\perp} / T_{\|}$, would find this point in the alfvenon area and could evolve into a soliton. This model for generation of mirror mode structures 
in the magnetosheath should be investigated further with simulation methods.

\section{Conclusions}

Using ion measurements on Cluster in the magnetosheath we have derived empirically the parameters of the polybaric pressure model described by Eqs. (4-5). This model appears to provide a versatile and realistic replacement for polytropic pressure models used in fluid analytical derivations and simulations schemes.

Using two-fluid model with polybaric pressure we have demonstrated that the classical mirror instability threshold represents a limiting case $M=0$ of a large area in the phase space $(M, \alpha)$ covered by unstable, exponentially varying solutions denoted as slow alfvenons. These alfvenons have speeds in the range $0-V_{A}$ and consist of three types of nonlinear structures: Alfvén solitons, magnetosonic solitons, and non-stationary, nonlinear waves.

Applying multi-spacecraft timing to Cluster measurements with large separation distances $(\sim 2000 \mathrm{~km})$ we have shown that mirror structures move with respect to the plasma with speed of up to $43 \mathrm{~km} / \mathrm{s}$ and are not consistent with structures being frozen in plasma flow. The propagating fronts exhibit radius of curvature $\sim 10000 \mathrm{~km}$, which still justifies use of plane approximation on scales up to $\sim 1000 \mathrm{~km}$. Comparison of the observed mirror structures with theoretical alfvenon solutions show that they have properties of magnetosonic solitons with respect to the shape, size, amplitude, polarization and speed.

The present model implies answers (mentioned briefly in Sect. 5) to currently discussed and regarded as unresolved problems of mirror mode physics, related to the generation mechanism, saturation, sizes, amplitudes, generation of magnetic peaks and dips, as well as appearance below the instability threshold.

\section{Appendix A}

The standard fluid equations containing the plasma momentum and the generalized Ohm's law with electron inertial terms, applied to 1-D propagation in the $x$ direction, in the stationary wave frame, lead to a set of equations for dimensionless variables, $n=N / N_{0}, \mathbf{b}=\mathbf{B} / B_{0}, \mathbf{v}=\mathbf{V} / V_{A}$ (e.g. Stasiewicz, 2005a). The normal momentum equation represents a balance of pressure components

$2 M^{2} n^{-1}+b^{2}+\beta n^{\gamma} b^{\kappa}+\beta A_{p} \cos ^{2} \alpha=\mathrm{const}$,

where $\beta=\beta_{i}+\beta_{e}=2 \mu_{0} p_{\perp 0} / B_{0}^{2}, A_{p}=n^{\gamma} b^{\kappa-2}\left(a_{p}-1\right)$, $M=(\omega-\boldsymbol{k} \cdot \boldsymbol{V}) / k V_{A}$ is the Alfvén Mach number in the reference system under consideration, $V_{A}=B_{0}\left(\mu_{0} N_{0} m_{i}\right)^{-1 / 2}$ is the Alfvén speed, and $\alpha$ is the propagation angle with respect to the magnetic field. The anisotropy parameter $a_{p}$ is given by Eq. (5). The transverse components of the momentum equation give

$v_{y}=\frac{b_{y}}{M_{\|}}\left(1-\frac{\beta}{2} A_{p}\right)$

$v_{z}=\frac{b_{z}}{M_{\|}}\left(1-\frac{\beta}{2} A_{p}\right)-\frac{b_{z 0}}{M_{\|}}\left(1-\frac{\beta}{2} A_{p 0}\right)$,

where $b_{z 0}=\sin \alpha$ and $A_{p 0}=a_{p 0}-1$. The generalized Ohm's law can be written in dimensionless form as

$$
\begin{gathered}
-R_{e} \frac{\partial}{\partial x}\left(\frac{1}{n} \frac{\partial b_{z}}{\partial x}\right)+\frac{1}{M_{\|}} \frac{\partial b_{y}}{\partial x}=\frac{n v_{z}}{M_{\|}}-b_{z}+n b_{z 0} \\
R_{e} \frac{\partial}{\partial x}\left(\frac{1}{n} \frac{\partial b_{y}}{\partial x}\right)+\frac{1}{M_{\|}} \frac{\partial b_{z}}{\partial x}=b_{y}-\frac{n v_{y}}{M_{\|}} .
\end{gathered}
$$

The length $x$ is expressed in units of $\lambda_{i}, R_{e}=m_{e} / m_{i}$, and we have assumed isotropy of the electron pressure. Linearization of these equations assuming perturbations $\propto \exp (k x)$ leads to the dispersion Eq. (10). Integration of these equations has been used to obtain soliton solutions presented in this paper. The electron inertia is not important in the $(M, \alpha)$ area of mirror structures, so the second order derivatives can be dropped to facilitate the integration. Region, where the solutions numerically diverge is denoted as "non-stationary waves" in Fig. 3.

Acknowledgements. The authors acknowledge use of Cluster data provided to the Cluster Active Archive by E. Lucek, PI for FGM, magnetic field experiment, and I. Dandouras, PI for CIS, ion spectrometer experiment.

Topical Editor I. A. Daglis thanks one anonymous referee for her/his help in evaluating this paper.

\section{References}

Abraham-Shrauner, B.: Propagation of hydromagnetic waves through an anisotropic plasma, J. Plasma Phys., 1, 361-378, 1967.

Baumgärtel, K., Sauer, K., and Dubinin, E.: Towards understanding magnetic holes: hybrid simulations, Geophys. Res. Lett., 30, 1761, doi:10.1029/2003GL017373, 2003.

Belmont, G. and Mazelle, C.: Polytropic indices in collisionless plasmas: Theory and measurements, J. Geophys. Res., 97, 83278336, 1992.

Borgogno, D., Passot, T., and Sulem, P. L.: Magnetic holes in plasmas close to the mirror instability, Nonlin. Processes Geophys., 14, 373-383, 2007,

http://www.nonlin-processes-geophys.net/14/373/2007/.

Califano, F., Hellinger, P., Kuznetsov, E., Passot, T., Sulem, P., and Travnicek, P.: Nonlinear, mirror mode dynamics: simulations and modelling, J. Geophys. Res., 113, A08219, doi: 10.1029/2007JA012898, 2008.

Cheng, C. Z. and Johnson, J.: A kinetic-fluid model, J. Geophys. Res., 104, 413-427, 1999.

Chew, G. F., Goldberger, M. L., and Low, E. F.: The Boltzmann equation and the one-fluid hydromagnetic equations in the absence of particle collisions, Proc. Roy. Soc. London, A236, 112$118,1956$. 
Chust, T. and Belmont, G.: Closure of fluid equations in collisionless magnetoplasmas, Phys. Plasmas, 13, 012506, doi: 10.1063/1.2138568, 2006.

Constantinescu, O. D., Glassmeier, K.-H., Treumann, R., and Fornacon, K.-H.: Magnetic mirror structures observed by Cluster in the magnetosheath, Geophys. Res. Lett., 30, 1802, doi: 10.1029/2003GL017313, 2003.

Génot, V., Budnik, E., Hellinger, P., Passot, T., Belmont, G., Trávnıček, P. M., Sulem, P.-L., Lucek, E., and Dandouras, I.: Mirror structures above and below the linear instability threshold: Cluster observations, fluid model and hybrid simulations, Ann. Geophys., 27, 601-615, 2009, http://www.ann-geophys.net/27/601/2009/.

Hasegawa, A.: Drift mirror instability in the magnetosphere, Phys. Fluids, 12, 2642, doi:10.1063/1.1692407, 1969.

Hau, L.-N. and Sonnerup, B. U.: On slow-mode waves in an anisotropic plasma, Geophys. Res. Lett., 64, 1763-1766, 1993.

Hau, L.-N., Wang, B.-J., and Teh, W.-L.: Slow mode waves and mirror instability in gyrotropic Hall magnetohydrodynamic model, Phys. Plasmas, 12, 122904, doi:10.1063/1.2141931, 2005.

Hellinger, P.: Comment on the linear mirror instability near threshold, Phys. Plasmas, 14, 082105, doi:10.1063/1.2768318, 2007.

Horbury, T. S., Lucek, E. A., Balogh, A., Dandouras, I., and Reme, H.: Motion and orientation of magnetic field dips and peaks in the terrestrial magnetosheath, J. Geophys. Res., 109, A09209, doi:10.1029/2003JA010237, 2004.

Johnson, J. R. and Cheng, C. Z.: Global structure of mirror modes in the magnetosheath, J. Geophys. Res., 102, 7179-7189, 1997.

Joy, S. P., Kivelson, M. G., Walker, R. J., Kurhana, K. K., Russell, C. T., and Peterson, W. R.: Mirror mode structures in the Jovian magnetosheath, J. Geophys. Res., 111, A12212, doi: 10.1029/2006JA011985, 2006.

Kaufmann, R., Horng, J. T., and Wolfe, A.: Large amplitude hydromagnetic waves in the inner magnetosheath, J. Geophys. Res., 75, 4666-4676, 1970.

Kivelson, M. G. and Southwood, D. J.: Mirror instability II, The mechanism of nonlinear saturation, J. Geophys. Res., 101, 17365-17371, 1996.

Knetter, T., Neubauer, F. M., Horbury, T., and Balogh, A.: Fourpoint discontinuity observations using Cluster magnetic field data: a statistical survey, J. Geophys. Res., 109, A06102, doi: 10.1029/2003JA010099, 2004.

Kuznetsov, E., Passot, T., and Sulem, P. L.: Dynamical model for nonlinear mirror modes near threshold, Phys. Rev. Lett., 98, 235003, doi:10.1103/PhysRevLett.98.235003, 2007.

Lucek, E. A., Constantinescu, D., Goldstein, M. L., Picket, J., Pincon, J. L., Sahraoui, F., Treumann, R. A., and Walker, S. N.: The magnetosheath, Space Sci. Rev., 118, 92-152, 2005.

McKenzie, J. F., Dubinin, E., Sauer, K., and Doyle, T. B.: The applications of the constants of motion to nonlinear stationary waves in complex plasmas: a unified fluid dynamic viewpoint, J. Plasma Phys., 70, 431, doi:10.1017/S0022377803002654, 2004.

Mjølhus, E.: Velocity-amplitue relationship and polarization in families of MHD solitary waves, Phys. Scr., T122, 135-153, 2006.

Pantellini, F. G.: A model of the formation of stable nonpropagating magnetic structures in the solar wind based on the nonlinear mirror instability, J. Geophys. Res., 103, 4789-4798, 1998.
Passot, T. and Sulem, P. L.: A Landau fluid model with finite Larmor radius effects for mirror mode dynamics, J. Geophys. Res., 11, A04203, doi:10.1029/2005JA011425, 2006.

Pokhotelov, O. A., Sagdeev, R. Z., Balikhin, M. A., and Treumann, R. A.: Mirror instability at finite ion-Larmor radius wavelengths, J. Geophys. Res., 109, A09213, doi:0.1029/2004JA010568, 2004.

Rème, H., Aoustin, C., Bosqued, J. M., et al.: First multispacecraft ion measurements in and near the Earth's magnetosphere with the identical Cluster ion spectrometry (CIS) experiment, Ann. Geophys., 19, 1303-1354, 2001, http://www.ann-geophys.net/19/1303/2001/.

Sauer, K., Dubinin, E., and McKenzie, J. F.: Solitons and oscillitons in multi-ion space plasmas, Nonlin. Processes Geophys., 10, 121-130, 2003, http://www.nonlin-processes-geophys.net/10/121/2003/.

Schwartz, S. J., Burgess, D., and Moses, J. J.: Low-frequency waves in the Earth's magnetosheath: present status, Ann. Geophys., 14, 1134-1150, 1996, http://www.ann-geophys.net/14/1134/1996/.

Snyder, P. B., Hammett, G. W., and Dorland, W.: Landau fluid models of collisionless magnetohydrodynamics, Phys. Plasmas., 4, 3974, doi:10.1063/1.872517, 1997.

Sonnerup, B. U. and Cahill, L. J.: Magnetopause structure and attitude from Explorer 12 observations, J. Geophys. Res., 72, 171183, 1967.

Soucek, J., Lucek, E., and Dandouras, I.: Properties of magnetosheath mirror modes observed by Cluster and their response to changes in plasma parameters, J. Geophys. Res., 113, A04203, doi:10.1029/2007JA012649, 2008.

Southwood, D. J. and Kivelson, M. G.: Mirror instability I, The physical mechanism of linear instability, J. Geophys. Res., 98, 9181-9187, 1993.

Stasiewicz, K.: Reinterpretation of mirror modes as trains of slow magnetosonic solitons, Geophys. Res. Lett., 31, L21804, doi:10. 1029/2004GL021282, 2004a.

Stasiewicz, K.: Theory and observations of slow-mode solitons in space plasmas, Phys. Rev. Lett., 93, 125004, doi:10.1103/ PhysRevLett.93.125004, 2004b.

Stasiewicz, K.: Nonlinear Alfvén, magnetosonic, sound, and electron inertial waves in fluid formalism, J. Geophys. Res., 110, A03220, doi:10.1029/2004JA010852, 2005a.

Stasiewicz, K.: Ion pressure equations derived from measurements in space, Phys. Rev. Lett., 95, 015004, doi:10.1103/PhysRevLett. 95.015004, 2005b.

Stasiewicz, K. and Ekeberg, J.: Dispersive MHD waves and alfvenons in charge non-neutral plasmas, Nonlin. Processes Geophys., 15, 681-693, 2008, http://www.nonlin-processes-geophys.net/15/681/2008/.

Tatrallyay, M., Erdös, G., Balogh, A., and Dandouras, I.: The evolution of mirror type magnetic fluctuations in the magnetosheath based on multipoint observations, Adv. Space Res., 41, 15371544, 2008.

Tsurutani, B. T., Smith, E. J., Anderson, R. R., Ogilvie, K. W., Scudder, J. D., Baker, D. N., and Bame, S. J.: Lion roars and nonoscillatory drift mirror waves in the magnetosheath, J. Geophys. Res., 87, 6060-6072, 1982. 